\title{
Asymmetry in Supernovae
}

\author{
Keiichi Maeda \\ Kavli Institute for the Physics and Mathematics of the Universe, University of Tokyo, \\ 5-1-5 Kashiwanoha, Kashiwa, Chiba 277-8583, Japan \\ email: keiichi.maeda@ipmu.jp
}

\begin{abstract}
Asymmetry in the innermost part of the supernova (SN) ejecta is a key to understanding their explosion mechanisms. Late-time spectroscopy is a powerful tool to investigate the issue. We show what kind of geometry is inferred for different types of $\mathrm{SNe}$ - core-collapse $\mathrm{SNe} \mathrm{Ib} / \mathrm{c}$, those associated with Gamma-Ray Bursts (GRBs), and thermonuclear SNe Ia -, and discuss implications for the explosion mechanisms, observational diversities, and cosmological applications. For $\mathrm{SNe} \mathrm{Ib} / \mathrm{c}$, the data show the clear deviation from spherical symmetry, and they are most consistent with the bipolar-type explosion as the characteristic geometry. Detailed modeling of optical emissions from SN 1998bw associated with GRB980425 indicates that this SN was in the extreme end of the bipolar explosion, suggesting that the explosion mechanisms of canonical SNe Ib/c and GRB-SNe are different. The situation is different for SNe Ia. Late-time spectra indicate the deviation from spherical symmetry, but for SNe Ia the explosion is asymmetric between two hemispheres, i.e., one-sided explosions. The diversities arising from different viewing directions can nicely explain (a part of) observational diversities of SNe Ia, and correcting this effect may improve the standard-candle calibration of SNe Ia for cosmology.
\end{abstract}

Keywords. supernovae: general - gamma rays: bursts

\section{Introduction}

Supernovae (SNe) are classified into several types by characteristics in their spectra around the maximum light (e.g., Filippenko 1997), and the spectral typing has been linked to the progenitor scenarios through the amount of the stellar envelope present at the time of explosion (e.g., Nomoto et al. 1995). Stars that retain their H envelope produce SNe with H-rich spectra, classified as SNe II. Stars that have lost at least a large fraction, if not all, of the $\mathrm{H}$ envelope produce He-rich $\mathrm{SNe} \mathrm{IIb}, \mathrm{H}$-deficient $\mathrm{SNe}$ $\mathrm{Ib}$, and both $\mathrm{H}$ and He-deficient $\mathrm{SNe} \mathrm{Ic}$, in order of increasing degree of the envelope stripping. They are believed to be an explosion of a massive star, i.e., core-collapse (CC) SNe. SNe Ia show neither $\mathrm{H}$ nor He, and they are further characterized by a strong Si absorption. The spectral characteristics of SNe Ia matches well the standard scenario that they are a result of a thermonuclear explosion of a white dwarf, reaching (nearly) the Chandrasekhar limiting mass (e.g., Nomoto et al. 1984; Woosley \& Weaver 1986).

Some SNe Ic ('broad-line SNe Ic') show broad absorption features, indicating a higher expansion velocity and a larger energy than other SNe $\left(E_{\mathrm{K}} \sim 10^{51} \mathrm{erg}\right.$; e.g., Iwamoto et al. 1998). The most energetic broad-line SNe Ic reach $E_{\mathrm{K}} \gtrsim 10^{52} \mathrm{erg}$, and some of them are associated with long-soft Gamma-Ray Bursts (hereafter GRBs for the long-soft class) as exemplified by GRB-SNe 1998bw and 2003dh (Galama et al. 1998; Hjorth et al. 2003). The association has been regarded as solid evidence for the popular scenario that GRBs are the outcome of the collapse of a stripped envelope $\mathrm{C}+\mathrm{O}$ star (e.g., Woosley 1993). A majority of SNe Ic are not associated with GRBs: clarifying the origin of the difference will shed light on the still-unresolved explosion mechanism(s) of both of them.

SNe Ia are extremely useful for cosmology (Permutter et al. 1999; Riess et al. 1998). 


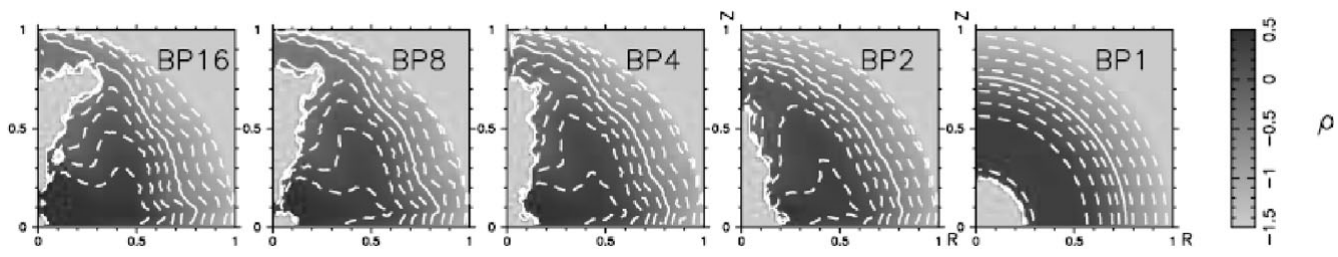

Figure 1. Examples of bipolar SN Ib/c explosion models. The degree of asphericity is expressed by a parameter BP in this model sequence, which is larger for more extremely bipolar explosions (Maeda et al. 2006a).

They are mature standardized candles, since their luminosity can be estimated through a phenomenological relation between the peak luminosity and the light curve decline rate (Phillips et al. 1999). Their maximum-light spectra however show diversities, highlighted by different absorption velocities and the speed of the velocity decrease (Benetti et al. 2005). This is likely related to details of the explosion process.

For both CC SNe (plus GRBs) and SNe Ia, the explosion mechanisms have not been clarified in detail. Recent simulations suggest that a key to understanding their explosion mechanisms is the geometry of the explosion. Different scenarios predict different types of geometry in the explosion, thus observationally deriving the geometry of the innermost part of SN ejecta is of highest importance. In this review, we summarize what has been learned through late-time spectroscopy of SNe about their explosion mechanisms.

\section{Possible explosion geometry and expected features}

The explosion mechanisms of both CC SNe and SNe Ia have not been clarified in detail. For CC SNe, recent theoretical studies have shown that the standard delayed neutrino heating mechanism requires significant deviation from spherical symmetry so that the process works effectively. The initial results in $2 \mathrm{D}$ simulations show the development of the bipolar-type explosion through the standing accretion shock instability (SASI; e.g., Blondin et al. 2003). It is not clear yet if this is an artifact of the imposed axisymmetry so far the agreement seems controversial (e.g., Blondin \& Mezzacappa 2007; Iwakami et al. 2009; Takiwaki et al. 2012). In addition to the SASI scenario, there are other possible mechanisms that can/should generate characteristic geometry in the explosion for example, if the magnetic field plays an important role (Takiwaki et al. 2009), as has been suggested for GRB-SNe and some CC SNe, it likely generates the bipolar-type structure. In $\mathrm{CC}$ SNe, the materials in the direction of the stronger shock suffer from the more energetic explosive nucleosynthesis, while in the other directions the pre-explosion composition will be preserved. We thus expect that the distribution of Fe (created as ${ }^{56} \mathrm{Ni}$ at the strong shock) follows the geometry of the explosion. Distribution of O (i.e., pre-SN composition) will reflect the explosion geometry as well, where the 'hole' in the $\mathrm{O}$ distribution indicates the strong shock wave in that direction. As an example, the bipolar explosion predicts that Fe is distributed along the axis of symmetry, while $\mathrm{O}$ is distributed as a 'torus' (Fig. 1: from Maeda et al. 2006a).

The possible importance of bulk asymmetry in SN Ia explosions has been recognized recently (e.g., Kasen et al. 2009; Maeda et al. 2010a). Within the standard delayed detonation scenario (Khokhlov 1991), where the explosion is first triggered by ignition of deflagration sparks near the center then energized further by the transition to the detonation wave, an issue is how the initial sparks are ignited. Perturbations within the progenitor, e.g., by convection, could naturally result in the off-set ignition (Kuhlen et al. 2006). If this is true, it is predicted that stable Fe-peak elements are produced by the 

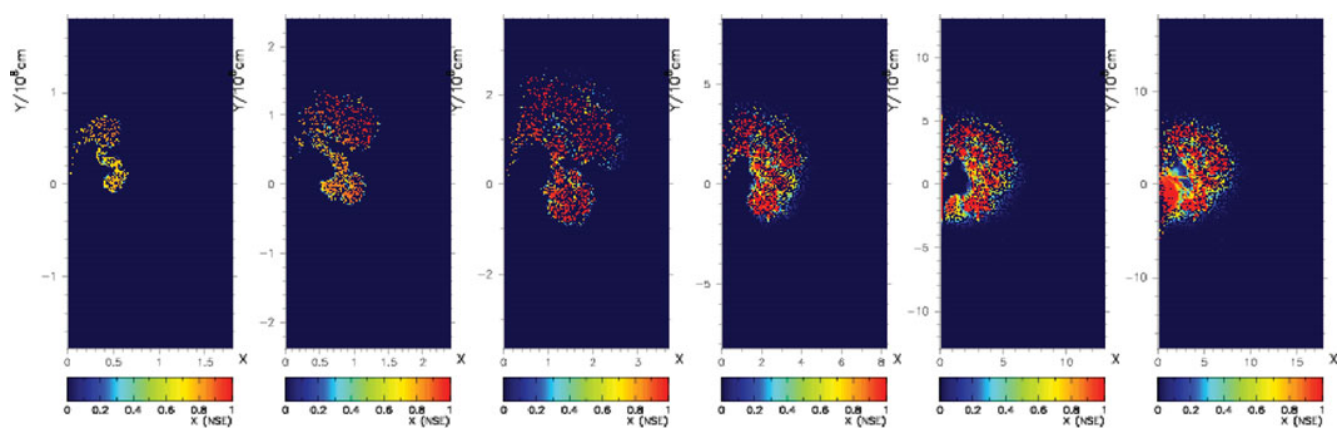

Figure 2. Temporal evolution of distribution of Fe-peaks (at 0.7, 0.9, 1.1, 1.3, 1.5, 1.7 seconds after the ignition, from left to right), in an offset delayed detonation model (Maeda et al. 2010b; see also Kasen et al. 2009). Shown here is the mass fraction of the Fe-peaks, from 0 (blue/dark) to 1 (red/bright). The deflagration phase (producing stable Fe-peaks) is highly asymmetric, while the detonation wave (producing ${ }^{56} \mathrm{Ni}$ ) expands nearly isotropically.

deflagration (including stable $\mathrm{Ni}$ ) at an off-set from the explosion center, while radioactive ${ }^{56} \mathrm{Ni}$ (which decays into Co then into $\mathrm{Fe}$ ) is produced by the detonation more or less in a spherically symmetric manner (e.g., Maeda et al. 2010b; Fig. 2). Other explosion models also predict large deviation from spherical symmetry; for example, the prompt detonation following the merger of two white dwarfs will be highly asymmetric (Röpke et al. 2012; Sim et al. 2012).

\section{Late-time spectroscopy to study explosion geometry}

As the SN ejecta expand, the ejecta become transparent at about 100 or 200 days after the explosion. Afterwards, the effect of the radiation transfer is minimal to alter the line profile. At this late epoch, the SN spectrum is nebular, showing emission lines mostly of forbidden transitions. Thanks to the homologous nature of the SN expansion, the Doppler shift indicates where the photon was emitted. A photon emitted from the near/far side of the ejecta is blueshifted/redshifted. Thus, the line profile can be used as a direct tracer of the distribution of the emitting materials within the SN ejecta.

The general behavior of the line profile for different distributions of material is model independent. If the distribution is spherical, then the center of the line should be at the rest wavelength of the transition, and the line profile should be symmetric between the blue and red. If the ejecta is axisymmetric and also symmetric with respect to the equator (including the bipolar distribution), the line profile should be symmetric with respect to the rest wavelength, but the peak position is generally different from the rest wavelength (e.g., double peaks). If the distribution is still axisymmetric but there is an imbalance between two hemispheres (i.e., a one-sided distribution), then the line neither has to peak at the rest wavelength, nor has to be symmetric.

\section{Asymmetry in $\mathrm{SNe} \mathrm{Ib} / \mathrm{c}$ and connection to GRBs}

Detailed study of the geometric nature of the explosion of $\mathrm{SNe} \mathrm{Ib} / \mathrm{c}$ has been initially developed for GRB-SN Ic 1998bw. SN Ic 1998bw was discovered in association with GRB 980425 (Galama et al. 1998). Modeling the early phase observations (through two or three months after the explosion) showed that it is a highly energetic explosion (Iwamoto et al. 1998). Its late-time spectra showed the velocity of Fe higher than O (Mazzali et al. 2001), and a highly bipolar explosion was suggested (Maeda et al. 2002). Meanwhile, its explosion geometry was intensively studied based on multi-D radiation 

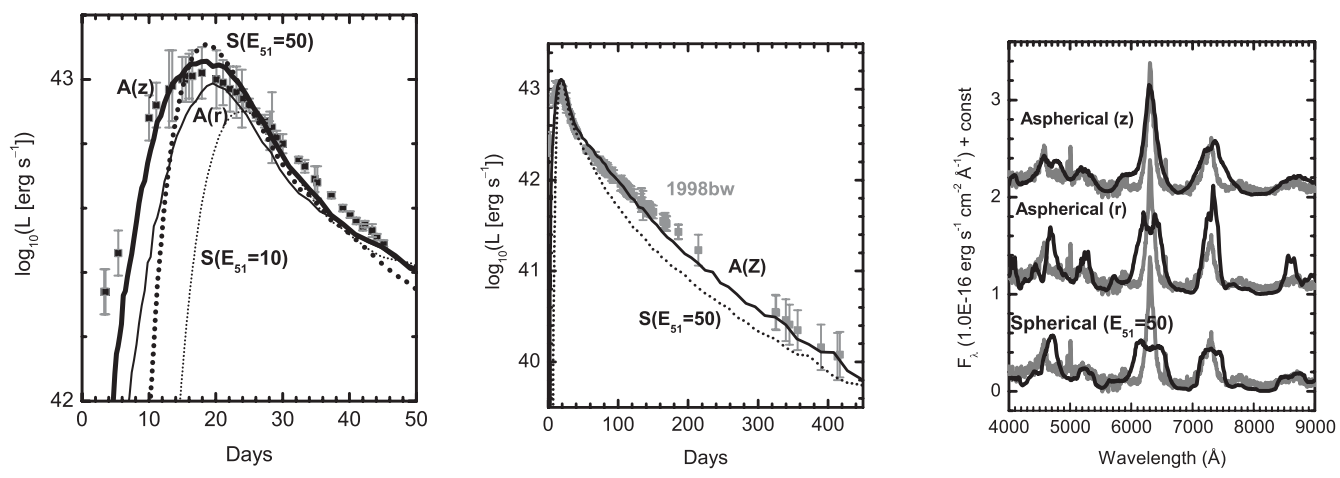

Figure 3. Radiation transfer models for GRB-SN 1998bw based on the bipolar model (Fig. 1). The left and middle panels show the bolometric light curve of SN 1998bw, as compared with the bipolar model BP8 (denoted by 'A') with $E_{\mathrm{K}}=2 \times 10^{52} \mathrm{erg}$ as seen on-axis ( $z$; thick solid) and off-axis ( $r$; thin solid), and the spherical model BP1 (denoted by ' $\mathrm{S}$ '; dotted lines). The right panel shows the late-time spectrum of SN 1998bw as compared with the model BP8 from two different directions and with the spherical model BP1.

transfer calculations (Fig. 3; Maeda et al. 2006a; Maeda et al. 2006b; Tanaka et al. 2007) applied to the observations of SN 1998bw. They concluded that no spherical models can consistently reproduce the observations from early through late phases, although a bipolar explosion model can reproduce these observations consistently. Within the framework of the bipolar explosion models, they showed that the parameters for the explosion can be constrained into a small parameter space: the main-sequence mass $M_{\mathrm{ms}} \sim 40 M_{\odot}$, the explosion energy $E_{\mathrm{K}} \sim 10-20 \times 10^{51}$ erg (smaller than the spherically symmetric estimate by a factor of a few), the degree of bipolarity BP $\sim 8-16$ (Fig. 1), and the viewing direction from the pole $\theta \sim 0-30^{\circ}$.

In $\mathrm{SNe} \mathrm{Ib} / \mathrm{c}, \mathrm{O}$ is the dominant element, and [OI] $\lambda \lambda 6300,6363$ is the strongest in their late-time spectra. Luckily, this wavelength range is free from contamination from other lines. Maeda et al. (2002) predicted that a bipolar explosion should produce double peaks in the $[\mathrm{OI}]$ if viewed from the equatorial direction as $\mathrm{O}$ is distributed in a torus - thus, if other SNe share a similar bipolar geometry with SN 1998bw, then there must be SNe showing the double peaks in the $[\mathrm{OI}]$ profile. Indeed, such a double peaked profile was discovered after the prediction, in the late-time spectrum of SN 2003jd - a broad-lined SN Ic similar to SN 1998bw, but without an associated GRB (Mazzali et al. 2005). This led us to suggest that SN 2003jd was similar to SN 1998bw but viewed off-axis.

We coordinated late-time spectroscopy of SNe Ib/c with Subaru and VLT telescopes, and investigated the profile of the $[\mathrm{OI}]$. Given that the line profile is only a function of the geometry and the viewing direction, the number of $\mathrm{SNe} \mathrm{Ib/c} \mathrm{that} \mathrm{show} \mathrm{the} \mathrm{doubly-}$ peaked [OI] line to the total number of $\mathrm{SNe} \mathrm{Ib} / \mathrm{c}$ sample should provide a measure for the typical degree of asphericity. Figure 4 shows the [OI] line profiles of $18 \mathrm{SNe} \mathrm{IIb/Ib/c}$ (Maeda et al. 2008). We found that the doubly-peaked profile is not rare, suggesting that the asphericity is a generic feature in SNe IIb/Ib/Ic (see also Modjaz et al. 2008; Taubenberger et al. 2009). As shown in Figure 4, the characteristic profile is either a single peak $(\mathrm{S})$ or double peaks $(\mathrm{D})$, and in both cases the line profile is rather symmetric with respect to the rest wavelength if the observation is performed at $\gtrsim 200$ days (see, however, Taubenberger et al. 2009). These features require the deviation from spherical symmetry, but the axisymmetry and the symmetry with respect to the equator should be roughly preserved - the straightforward interpretation is a bipolar-type geometry. Quantitatively, the fraction of SNe showing the doubly-peaked [OI] is $39 \pm 11 \%$. This indicates that the 


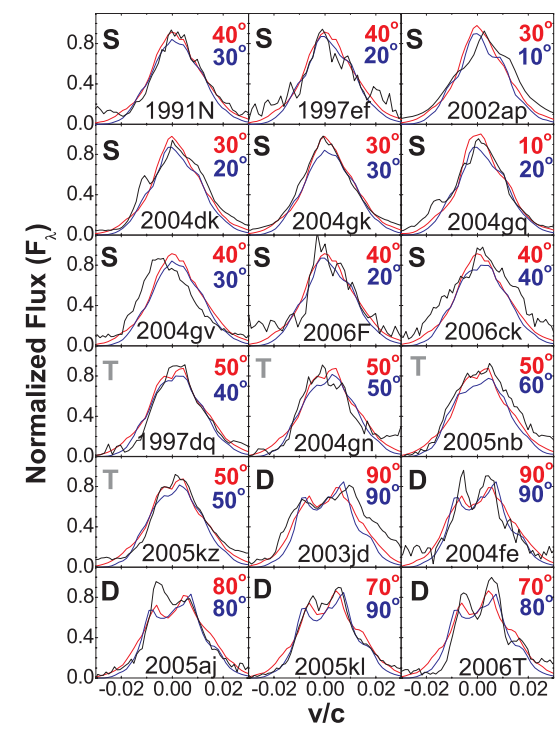

Figure 4. The line profile of $[\mathrm{OI}]$ in late-time spectra of $18 \mathrm{SNe} \mathrm{IIb} / \mathrm{Ib} / \mathrm{Ic}$ (black). Also shown are the synthetic line profiles for models BP8 and BP2 (see Fig. 1).

typical degree of the asphericity is moderate, i.e., $\mathrm{BP}=2$ (Fig. 1). This is smaller than the degree of asphericity derived for SN 1998bw $(\mathrm{BP}=8-16)$.

\section{Asymmetry in SNe Ia and cosmological application}

The offset ignition model (Fig. 2) predicts that an emission from the deflagration ash (showing an offset) should show a variation in the wavelength for different viewing directions, while that from the detonation ash (spherically distributed) should show little variation (Maeda et al. 2010b). We have investigated emission processes within the SN Ia ejecta in late-phases (Maeda et al. 2010c; see also Mazzali et al. 2007), and pointed out that the [Fe III] blend at $4,700 \AA$ is emitted from the detonation ash while $[\mathrm{Fe}$ II $] \lambda 7155$, $[\mathrm{Ni}$ II $] \lambda 7378$, and some NIR [Fe II] lines are emitted from the deflagration ash.

This analysis allowed us to investigate the explosion geometry though the existing

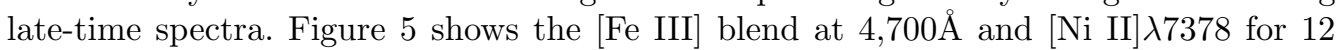
SNe Ia. The [Fe III] blend does not show significant shift for all SNe Ia. On the other hand, we discovered that $[\mathrm{Ni} \mathrm{II}] \lambda 7378$ does show variations in its central wavelength for different SNe Ia; some SNe show redshift and others blueshift. Also, the wavelength of [Ni II] $\lambda 7378$ does not show significant temporal evolution when late-time spectra at multiple epochs are available. These observational behaviors reject the possibility that the shift is produced by non-complete transparency or by contaminations from other lines.

Although the above observations indicate that an asymmetric, offset ignition is a generic feature of SNe Ia, the argument is based on a few lines only. This can be further tested for individual SNe for which late-time spectra are obtained with wide wavelength coverage, using various lines. This test has been performed for SN 2003hv. We have found that the lines in its late-time optical through mid-IR spectrum can indeed be divided into two groups, one showing a blueshift (with similar degree) and the other showing virtually no shift, and that grouping into these two categories is fully consistent with the above expectation. A model introducing the offset velocity of $\sim 3,500 \mathrm{~km} \mathrm{~s}^{-1}$ reproduces all the emission lines, without other parameters invoked to explain these different behaviors for different lines (Maeda et al. 2010c). 

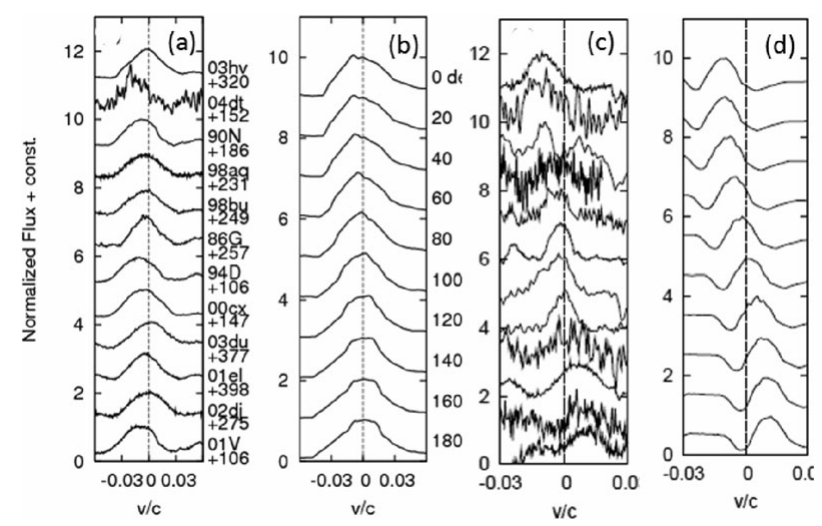

Figure 5. Late-time spectra of SNe Ia (Maeda et al. 2010c). (a) Observed and (b) model line profiles of [Fe III] blend at $4700 \AA$ and (c) observed and (d) model line profiles of [Ni II] $\lambda 7378$. The model profiles are shown for various viewing directions.

The finding has implications for explaining observational diversity and the cosmological applications. Spectral properties of SNe Ia are not uniform despite the standardized candle nature in the light curve (Benetti et al. 2005). In the early photospheric phase, materials moving toward the observer produce blueshifted absorption lines. The absorption velocity decreases, following the recession of the photosphere. The velocity gradient $\left(\dot{v}_{\mathrm{Si}}\right)$ is defined as a rate of the decrease in the Si II $\lambda 6355$ absorption velocity. SNe that show $\dot{v}_{\mathrm{Si}}>70 \mathrm{~km} \mathrm{~s}^{-1}$ day $^{-1}$ are classified as the high velocity gradient (HVG) group, while those showing smaller $\dot{v}_{\mathrm{Si}}$ are called the low velocity gradient (LVG) SNe Ia. It has been shown that $\dot{v}_{\mathrm{Si}}$ is not correlated with the decline rate. In addition, SNe Ia show a variation in their peak colors (e.g., Folatelli et al. 2010). The origin of these diversities beyond the 'one-parameter' description (by the decline rate) has not yet been clarified.

If the explosion is asymmetric, it raises an interesting question - whether the observed diversities could be related to the diversities arising from the explosion asymmetry and different viewing directions. Figure 6 provides a comparison between the velocity gradient $\left(\dot{v}_{\mathrm{Si}}\right)$ and the late-time emission-line velocity shift $\left(v_{\text {neb }}\right.$; measured by the Doppler shift of $[\mathrm{Fe} \mathrm{II}] \lambda 7155$ and [Ni II] $\lambda 7378$ ) as investigated by Maeda et al. 2010a). These two quantities turned out to be related, with HVG SNe showing redshift in late-time spectra. The correlation indicates that there is no intrinsic difference in HVG and LVG SNe, but the different appearance is merely a consequence of different viewing directions.

SNe Ia show a variation in their peak $B-V$ color. This is another major issue in SN cosmology since the estimate of extinction (thus distance) relies on the intrinsic color of SNe Ia. By comparing the 'viewing direction' and the color of SNe Ia, we have shown that that the intrinsic color variation is at the level of $B-V \sim 0.2 \mathrm{mag}$, and the variation within this level can be attributed to the difference arising from different viewing directions (Maeda et al. 2011).

\section{Conclusions}

In this review, we discussed how the geometry in the SN explosions has been obtained through the late-time spectroscopy. Our findings are summarized as follows.

- SNe Ib/c: The late-time [OI] profiles show the deviation from spherically symmetric explosions. A straightforward interpretation is that the characteristic geometry is (moderately) bipolar-like. 


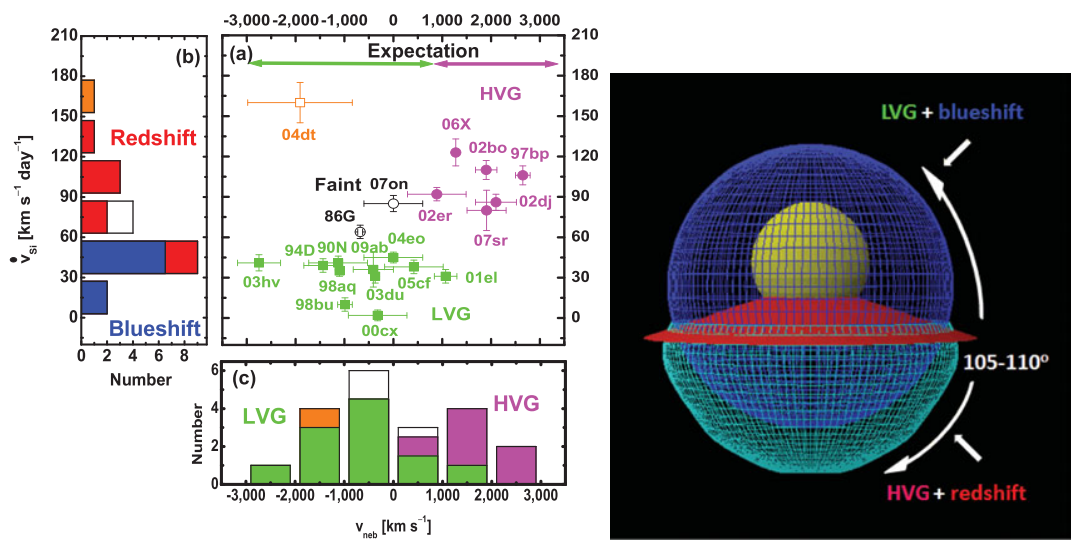

Figure 6. The left panel shows a comparison between the velocity gradient $\left(\dot{v}_{\mathrm{Si}}\right)$ and the latetime emission line velocity shift $\left(v_{\text {neb }}\right)$ (Maeda et al. 2010a). The right panel shows a schematic picture of SN Ia ejecta derived from the late-time spectra and its relation to the velocity gradients. Depending on the viewing direction an SN appears either as HVG or LVG in this scenario.

- GRB-SN 1998bw: Detailed radiation transfer modeling coupled with the late-time spectrum analysis indicates a bipolar explosion with a more extreme degree of asymmetry than in other $\mathrm{SNe} \mathrm{Ib} / \mathrm{c}$. This supports the idea that the explosion mechanism of GRBassociated broad-line SNe Ic is different from canonical SNe Ib/c. Also, the energy derived for the bipolar explosion model is a factor of a few smaller than the value obtained assuming a spherically symmetric explosion, and this effect must be taken into account in considering the explosion mechanism and energetics.

- SNe Ia: Late-time spectrum analysis suggests that the explosion has a bulk offset in its mechanism. Distribution of different isotopes are consistent with the expectation from the delayed detonation model, but further study is necessary to investigate if different explosion mechanisms can explain the behavior as well. In any case, correlations between the late-time emission line velocity shift and other observables in the early phases (i.e., velocity gradient, $B-V$ color) suggest that the combination of the asymmetric explosion and various viewing directions is a source of at least a part of observational diversities of SNe Ia. Understanding this effect is important for future use of SNe in cosmology.

\section{Acknowledgements}

This research is supported by World Premier International Research Center Initiative (WPI Initiative), MEXT, Japan and by Grant-in-Aid for Scientific Research (23740141).

\section{References}

Benetti, S., et al. 2005, ApJ, 623, 1011

Blondin, J. M., Mezzacappa, A., \& DeMarino, C. ApJ, 584, 971

Blondin, J. M. \& Mezzacappa, A. 2007, Nature, 445, 58

Filippenko, A. V. 1997, ARAA, 35, 309

Folatelli, G., et al. 2010, AJ, 139, 120

Galama, T. J., et al. 1998, Nature, 395, 670

Hjorth, J., et al. 2003, Nature, 423, 847

Iwakami, W., et al. 2009, ApJ, 700, 232

Iwamoto, K., et al. 1998, Nature, 395, 672

Kasen, D., Röpke, F., \& Woosley, S. E. 2009, Nature, 460, 869

Khokhlov, A. M. 1991, A\& A, 245, 114 
Kuhlen, M., Woosley, S. E., \& Glatznaier, G. A. 2006, ApJ, 640, 407

Maeda, K., et al. 2002, ApJ, 565, 405

Maeda, K., et al. 2006a, ApJ, 640, 854

Maeda, K., Mazzali, P. A., \& Nomoto, K. 2006b, ApJ, 645, 1331

Maeda, K., et al. 2008, Science, 319, 1220

Maeda, K., et al. 2010a, Nature, 466, 82

Maeda, K., et al. 2010b, ApJ, 712, 624

Maeda, K., et al. 2010c, ApJ, 708, 1703

Maeda, K., et al. 2011, MNRAS, 413, 3075

Mazzali, P. A., et al. 2001, ApJ, 559, 1047

Mazzali, P. A., et al. 2005, Science, 308, 1284

Mazzali, P. A., et al. 2007, Science, 315, 825

Modjaz, M., et al. 2008, ApJ, 687, L9

Nomoto, K., Thielemann, F.-K., \& Yokoi, K. 1984, ApJ, 286, 644

Nomoto, K., Iwamoto, K., \& Suzuki, T. 1995, Phys. Rep., 256, 173

Permutter, S., et al. 1999, ApJ, 517, 565

Phillips, M. M., et al. 1999, AJ, 118, 1766

Riess, A., et al. 1998, AJ, 116, 1009

Röpke, F. K., et al. 2012, ApJ, 750, 19

Sim, S., et al. 2012, MNRAS, 420, 3003

Takiwaki, T., Kotake, K., \& Sato, K. 2009, ApJ, 691, 1360

Takiwaki, T., Kotake, K., \& Suwa, Y. 2012, ApJ, 749, 98

Tanaka, M., et al. 2007, ApJ, 668, L19

Taubenberger, S., et al. 2009, MNRAS, 397, 677

Woosley, S. E. \& Weaver, T. A. 1986, ARAA, 24, 205

Woosley, S. E. 1993, ApJ, 405, 273

\section{Discussion}

Moiseenko: Can strictly spherically symmetrical supernovae exist in nature?

MAEDA: There is probably no 'strictly' spherical explosions in nature. SNe of different types show different degrees of deviation from spherical symmetry, for example this is more extreme in SNe Ib/c than SNe Ia as we see in the data, and there may be a class of SNe that are less asymmetric than those we have analyzed. Among the proposed explosion mechanisms, pair-instability SNe do not require the asymmetry in its mechanism, and they seem to be a candidate of a more or less spherically symmetric explosion.

MoDJAZ: For SNe Ib/c, there is a worry for $[\mathrm{OI}] \lambda \lambda 6300,6364$ that its doublet nature potentially produces the double peaks. Are you planning to include the radiative transfer models for other lines, e.g., OI $\lambda 7774$, Ca II?

MAEDA: In my personal view point, the argument for explaining the [OI] double peaks by the doublet nature has not been well justified. The center of the observed double peaks is close to $6300 \AA$ in the late phases or even bluer in the earlier phases, while the doublet center should be redder than $6300 \AA$. Thus the interpretation requires one to introduce artificially very high optical depths. However, such an effect is not seen in SNe II where the doublet is clearly detected separately, despite the expectation that SNe II are more dense than $\mathrm{SNe} \mathrm{Ib} / \mathrm{c}$ and such an effect, if it exists, should be clearly seen. In any case, it is very important and interesting to look into other lines as an independent check for the geometrical interpretation. Yes, we are planning to provide more detailed radiation transfer models to make the full analysis of the spectra, not only for the geometry study but also for studying other properties of SNe as well. 